\title{
COMUNIDADE INFESTANTE NO CONSÓRCIO DE MILHO COM LEGUMINOSAS ${ }^{1}$
}

\author{
DAGOBERTO MARTINS ${ }^{2}$
}

RESUMO

\begin{abstract}
Estudou-se o consórcio de milho com adubos verdes: Cajanus cajam, Stizolobium aterrimum, Crotalaria spectabilis e Dolichos lablab, e avaliou-se os efeitos das relações entre comunidade infestante, milho e leguminosas. Os tratamentos estudados foram: (i) milho em monocultivo mantido no limpo; (ii) milho em monocultivo mantido no mato; (iii) consórcio de milho com leguminosas em semeadura simultânea; (iv) cultivo consorciado de milho com leguminosas semeadas 21 dias após o milho. Brachiaria plantaginea foi a principal planta daninha da área experimental. A produção de matéria seca pelas plantas de milho foi afetada pela presença das plantas daninhas e leguminosas quando a semeadura do milho e leguminosas foi simultânea. A produção de matéria seca pelas leguminosas foi maior quando semeadas 21 dias após o milho. C. espectabilis foi a leguminosa menos
\end{abstract}

apta para conviver no consórcio. Stizolobium aterrimum foi a leguminosa com maior produção de matéria seca, nos dois sistemas de consórcio, a que menos afe tou as plantas de milho e, quando semeada simultaneamente ao milho reduziu a densidade de plantas daninhas em relação ao milho em monocultivo. A altura de plantas, a altura de inserção da primeira espiga e a produtividade de milho foram reduzidas pela presença de plantas daninhas e leguminosas quando a semeadura foi simultânea à do milho. As leguminosas reduziram a população de plantas daninhas sem afetar as plantas de milho e nem sua produtividade, quando semeadas 21 dias após o milho.

Palavras-chave: Adubo verde, interferência, Brachiaria plantaginea, Crotalaria spectabilis, Stilozobium aterrimum, Cajanus cajam, Dolichos lablab.

\section{ABSTRACT \\ The weed interference effects of intercropping legume species with corn}

This research was undertaken to study the effects of intercropping some legume species with corn on the weed community and vice-versa. Cajanus cajan, Stilozobium aterrimum, Crotalaria spectabilis and Dolichos lablab were used as green legumes. The trial was carried out in the Cascavel region, Paraná state, Brazil. Monocropping corn weed-free and monocropping corn without weeding were compared with corn + green legumes intercropped and sowed at the same time, and corn + green legumes intercropped (legumes sowed 21 days after corn sowing). Brachiaria plantaginea was the main weed species in the experimental area. Corn shoot dry weight was reduced by weeds and green legumes when the component crops were sowed at the same time. Legumes shoot dry weight was higher when sowed 21 days after sowing corn. C. espectabilis was the worst green legume in the intercropping system and the Stilozobium aterrimum was the best one. The green legumes when sowed 21 days after corn reduced the weed infestation without affecting the corn plants or the corn grain yield.

Additional index words: Brachiaria plantaginea, Crotalaria spectabilis, Stilozobium aterrimum, Cajanus cajam, Dolichos lablab.

\footnotetext{
${ }^{1}$ Recebido para publicação em 01/07/94 e na forma revisada em 31/12/94

${ }^{2}$ Professor Assistente, Doutor, FCA/UNESP, Caixa Postal 237, Botucatu, SP 18603-970
} 


\section{INTRODUÇÃO}

O consórcio de culturas é uma prática agrícola que visa o estabelecimento de mais de uma espécie em uma mesma área. Para isto, é necessário que existam efeitos benéficos ou não prejudiciais de uma cultura sobre a outra (Yadav, 1981; Pandey \& Pendeleton, 1986 e Ramakrishna et al., 1992), incremento da fertilidade do solo para culturas em sucessão (Nair et al., 1979; Bhardwaj et al., 1981; Yadav, 1981 e Kumar Rao et al., 1983), e arranjos populacionais adequados (Reis et al., 1985 e Lima et al., 1987).

Os estudos de consórcio envolvem quase que exclusivamente gramíneas e leguminosas, predominando entre as gramíneas o arroz (Bhardwaj et al., 1981 e Ramakrishna et al., 1992) e principalmente o milho (Dalai, 1974; Yadav, 1981; Silva Neto, 1981; Machado et al., 1984; Ramalho et al., 1984; Reis et al., 1985; Pandey \& Pendleton, 1986; Flesch \& Espindula, 1987; Lima et a1., 1987; Carvalho, 1990 e Chowdhury \& Rosario, 1992).

As leguminosas são uma fonte de nitrogênio menos dispendiosa, pois normalmente as espécies desta família apresentam simbiose com bactérias fixadoras de nitrogênio. $\mathrm{O}$ consórcio das leguminosas com o milho, além de melhorar a fertilidade do solo, pode ter em vista a nutrição animal, uma vez que a resteva do milho enriquecida com leguminosas proporciona uma alimentação mais nutritiva e palatável.

O comportamento das culturas no sistema de consórcío é diverso do encontrado no monocultivo, e as relações de interferência que se estabelecem com a comunidade infestante também modificam-se. Pesquisas sobre a interferência da comunidade infestante em consórcio de culturas são encontrados em número reduzido no Brasil (Silva Neto, 1981; Fleck et al., 1984 e Ramalho et al., 1989). Assim, o objetivo deste trabalho é estudar o consórcio de milho com leguminosas cultivadas como adubos verdes: feijão-guandú, mucunapreta, crotalária e labe-labe semeadas em duas épocas, e avaliar os efeitos das relações entre comunidade infestante, milho e leguminosas.

\section{MATERIAL E MÉTODOS}

O experimento foi instalado e conduzido na estação experimental da OCEPAR, em Cascavel/PR, no ano agrícola de 1989/90. O solo onde foi instalado o experimento é classificado como Latossolo Roxo distrófico, textura argilosa e apresentava: $\mathrm{pH}(\mathrm{CaC} 12)=4,9 ; 2,83 \%$ de carbono; 4,5 ppm de P; 0,15 m.eq de K; 5,5 m.eq de $\mathrm{Ca}+\mathrm{Mg} ; 4,25$ m.eq de $\mathrm{Ca}$ e 4,88 m.eq de $\mathrm{H}+\mathrm{Al}$.

Adotou-se o delineamento experimental de blocos casualizados, com três repetições. Os tratamentos estudados foram: (i) milho em monocultivo mantido no limpo; (ii) milho em monocultivo mantido no mato; (iii) consórcio de milho com leguminosas em semeadura simultânea; (iv) cultivo consorciado de milho com leguminosas semeadas 21 dias após o milho. As leguminosas utilizadas foram: feijãoguandú (Cajanus cajam L.), mucuna-preta (Stizolobium aterrimum Piper \& Tracy), crotalária (Crotalaria spectabilis Roth.) e labe-labe (Dolichos lablab L.). Nos tratamentos em consórcio não foi efetuado controle das plantas daninhas.
Em parcelas de 6,0 × 6,0 m, o milho (variedade OCEPAR 202) foi semeado no espaçamento de $1,0 \mathrm{~m}$. Nos sistemas consorciados, nas entrelinhas de milho, semeou-se duas linhas de leguminosa espaçadas de $0,5 \mathrm{~m}$ e distantes $0,25 \mathrm{~m}$ da linha de milho. A área útil da parcela foi composta pelas quatro linhas centrais de milho, desconsiderando-se $0,5 \mathrm{~m}$ de cada extremidade.

A semeadura do milho e leguminosas, em semeadura simultânea, fez-se no terceiro decêndio de outubro. A semeadura das leguminosas, no sistema consorciado, em semeadura não simultânea, fez-se 21 dias após a semeadura do milho.

$\mathrm{O}$ estande de milho foi de cinco plantas/m e o da mucuna-préta, labe-labe, feijão-guandú e crotalária de 10, 10,12 e 15 plantas $/ \mathrm{m}$, respectivamente. Para tal, foi efetuado um desbaste uma semana após a germinação de cada espécie. O preparo do solo foi efetuado no sistema convencional, com uma aração e duas gradagens. Tanto a semeadura do milho como a das leguminosas foi realizado de forma manual.

A adubação de plantio foi realizada somente para a cultura do milho com $380 \mathrm{~kg} / \mathrm{ha}$ da fórmula 4-14-8. Todas as parcelas receberam adubação de cobertura com uréia na dosagem de $40 \mathrm{~kg}$ de N/ha, aos 40 dias após a semeadura do milho. A adubação de cobertura foi feita de forma manual, colocando-se o fertilizante lateralmente às linhas de milho, na superfície.

Para determinação do peso de matéria seca das plantas cultivadas, retirou-se na área útil, cinco plantas de milho e cinco de leguminosas, de forma aleatória, aos 21, 42 e 63 dias após a semeadura das espécies. O mesmo foi efetuado para as leguminosas semeadas 21 dias após a semeadura do milho. A comunidade infestante foi avaliada aos 77 dias após a semeadura do milho através de duas amostragens de 0,25 $\mathrm{m}^{2} \mathrm{em}$ cada parcela. As plantas daninhas foram, identificadas, contadas e cortadas rente ao solo, utilizando-se apenas a parte aérea para determinação do peso de matéria seca.

Utilizou-se uma estufa de circulação forçada de ar, a $65-70^{\circ} \mathrm{C}$, para secarem as plantas de milho, leguminosas e infestantes, após o que foram pesadas.

$\mathrm{Na}$ colheita, realizada no terceiro decêndio de abríl, determinourse em cada parcela a altura de plantas, a altura de inserção da primeira espiga e o comprimento das espigas, através de 20 plantas. O rendimento em grãos foi obtido com a colheita da área útil das parcelas, e padronizou-se os resultados a $13 \%$ de umidade.

Os dados foram submetidos à análise de variância pelo Teste $\mathrm{F}$ e as médias dos tratamentos foram comparadas pelo Teste de Tukey $(\mathrm{P}>0,05)$.

\section{RESULTADOS E DISCUSSÃO}

Ocorreram na área experimental, Brachiaria plantaginea (capim-marmelada), Digitaria horizontalis (capim-colchão) e Bidens pilosa (picão-preto).

O capim-marmelada foi a espécie principal, tanto em número como em peso de matéria seca, tendo ocorrido de forma uniforme em todas as parcelas. A biomassa seca da parte aérea de suas plantas correspondeu a 97,4\% da comunidade infestante. 
O capim-colchão ocorreu somente nas parcelas onde a semeadura das leguminosas foi efetuada 21 dias após a do milho e, em apenas uma repetição de cada tratamento, em um total de sete plantas, representando $2,5 \%$ do total da matéria seca determinada. O picão-preto apareceu em uma única parcela do consórcio de milho com mucuna-preta semeada 21 dias após o milho. Suas plantas representaram 0,04\% do total da biomassa seca da comunidade infestante.

A diversidade de espécies que foi observada nas parcelas em que as leguminosas foram semeadas 21 dias após o milho pode ter sido provocada pela abertura dos sulcos nas entrelinhas do milho, para a semeadura das leguminosas, o que expôs novas camadas de solo, possibilitando a germinação de sementes trazidas à superfície, como o capim-colchão e o picão-preto, embora em quantidade reduzida. Estas plantas daninhas podem ter germinado junto com a cultura do milho, sendo eliminadas posteriormente pela competição exercida pelo capim-marmelada, que predominou em toda área experimental.

Em estudos anteriores, de consórcio de culturas, observaram-se comunidades infestantes compostas basicamente de espécies de folhas largas e não de gramíneas, como na presente pesquisa. Fleck et al. (1984) observaram que o caruru (Amaranthus spp) foi a principal planta daninha da comunidade infestante, compondo quase o total da população. Ramalho et al. (1989) verificaram que Blainvillea rhomboidea foi a planta daninha predominante, compondo $28 \%$ da população.

A predominância do capim-marmelada na área experimental está ligada à agressividade da espécie, que apresenta rápida emergência e resistência ao sombreamento. Estas características facilitam o estabelecimento desta espécie que pode formar um grande dossel de folhas acima de outras espécies.

Outro fator de importância relevante é o $\mathrm{pH}$ baixo do solo da área experimental, o que favorece o crescimento e desenvolvimento de B. plantaginea (Martins \& Pitelli, 1993) em detrimento da cultura.

A população de plantas daninhas, expressa pela densidade e produção de matéria seca da parte aérea (Tabela 1), foi menor no sistema consorciado em que o milho e leguminosas foram semeadas em épocas diferentes. Isto, explica-se pela eliminação de grande parte da população já estabelecida, por ocasião da abertura dos sulcos, nas entrelinhas do milho, para a semeadura das leguminosas, aos 21 dias após a semeadura do milho. Além disso, não houve novo fluxo de emergência de plantas daninhas.

A presença de feijão-guandú, mucuna-preta e labelabe, no sistema de semeadura simultânea a do milho, não reduziu a população de plantas daninhas (Tabela 1). Ao contrário, Silva Neto (1981), trabalhando com as mesmas espécies e sistema de consorciação, observou que essas leguminosas reduziram à população de plantas daninhas. Em trabalho semelhante, Fleck et al. (1984) observaram que a biomassa seca da comunidade infestante no milho consorciado com girassol e feijão, em semeadura simultânea, foi menor que no milho em monocultivo. A diferença entre o resultado obtido e o mencionado na literatura justifica-se pela composição da comunidade infestante. Em Cascavel/ PR, a população era formada predominantemente por gramí neas, enquanto nos trabalhos citados, por espécies de planta de folhas largas.

Nos dois sistemas de consorciação, não houve efeito diferenciado entre as leguminosas sobre a comunidade infestante, mas a mucuna-preta, em semeadura simultânea ao milho, reduziu a densidade de plantas daninhas em relação ao monocultivo do milho (Tabela 1) e favoreceu a produção de grãos desta cultura (Tabela 2).

A ocorrência de uma população de plantas daninhas menor, no consórcio com semeadura em épocas diferentes, explica-se pela eliminação de grande parte da população já estabelecida, por ocasião da abertura dos sulcos para a semeadura das leguminosas, aos 21 dias depois da semeadura do milho. Além disso, não houve um novo fluxo de emergência de plantas.

A crotalária não suportou a interferência das plantas daninhas e do milho, sendo eliminada quando semeada simultaneamente ao milho, produzindo as menores quantidades de matéria seca, quando semeada 21 dias após o milho (Tabela 1). A produção de matéria seca pelas outras leguminosas foi sempre maior quando semeadas 21 dias após o milho (Tabela 1).

A biomassa seca das plantas de milho, nos tratamentos com semeadura simultânea, foi reduzida pela interferência das leguminosas e comunidade infestante. Neste caso a mucuna-preta foi a que causou efeito menos intenso. Não houve decréscimos significativos na biomassa seca do milho, quando a semeadura das leguminosas foi realizada 21 dias após a semeadura do milho (Tabela 1 ).

Silva Neto (1981) não observou decréscimos na biomassa seca de milho quando este foi semeado simultaneamente com labe-labe e feijão-guandú. Esse resultado devese, talvez, à baixa pressão competitiva da comunidade infestante, pois não houve diferença entre a produção do milho mantido em monocultivo no limpo e no mato. Ao contrário, no trabalho ora em estudo, houve redução de $76 \%$ na produção de matéria seca do milho mantido no mato em relação ao mantido no limpo, aos 63 dias (Tabela 1).

O milho em monocultivo, mantido no mato apresentou produção de matéria seca menor que nos dois sistemas de consórcio, o que evidencia que as leguminosas auxiliaram no controle das plantas daninhas, destacando-se a mucuna-preta em semeadura simultânea ao milho (Tabela 1).

Nas duas épocas de semeadura a mucuna-preta foi a espécie que apresentou maior produção de matéria seca, seguida do labe-labe, feijão-guandú e crotalária. Estes resultados assemelham-se aos de Silva Neto (1981) que observou, nas mesmas épocas de avaliação, que o labe-labe foi a leguminosa com maior produção de biomassa seca, seguido da mucuna-preta e feijão-guandú. Este desempenho diferenciado das espécies leguminosas pode ser atribuído às comunidades infestantes e as condições edafo-climáticas diversas das regiões de São Paulo e Paraná, onde foram conduzidos os experimentos.

Do mesmo modo que no trabalho de Silva Neto (1981), o peso de 100 grãos não foi afetado pelos tratamentos (Tabela 2). Provavelmente, essa variável é característica dependente da variedade e pouco influenciado pelo meio.

De modo geral, comunidade infestante e as leguminosas não tiveram um efeito acentuado sobre o tamanho de 
TABELA 1 - Matéria seca de milho e leguminosas no período inicial de desenvolvimento e da comunidade infestante aos 77 dias após a semeadura do milho. Cascavel/PR, 1989/90.

\begin{tabular}{|c|c|c|c|c|c|c|c|c|}
\hline \multirow{3}{*}{ Tratamento } & \multicolumn{2}{|c|}{ Comunidade Infestante } & \multicolumn{6}{|c|}{ Matéria Seca } \\
\hline & \multirow{2}{*}{$\begin{array}{c}\text { Densidade } \\
\left(\mathrm{n}^{2} \text { ptas } / \mathbf{0 , 5} \mathbf{~ m}^{2}\right)\end{array}$} & \multirow{2}{*}{$\begin{array}{c}\text { Matéria } \\
\text { seca } \\
\left(\mathrm{g} / \mathbf{0 , 5} \mathrm{m}^{2}\right)\end{array}$} & \multicolumn{3}{|c|}{ Leguminosas (g) } & \multicolumn{3}{|c|}{ Milho (g) } \\
\hline & & & 21 dias & 42 dias & 63 dias & 21 dias & 42 dias & 63 dias \\
\hline 1. Milho no limpo & - & - & - & - & - & $14,6 \mathrm{~A}$ & $194,4 \mathrm{~A}$ & $293,3 \mathrm{~A}$ \\
\hline 2. Milho no mato & $106,2 \mathrm{~A}$ & $201,1 \mathrm{~A}$ & - & - & - & $11,3 \mathrm{~B}$ & $43,3 \mathrm{E}$ & $70,7 \mathrm{C}$ \\
\hline \multicolumn{9}{|l|}{ Semeadura Simultânea } \\
\hline 3. Milho + Crotalária & $81,3 \mathrm{AB}$ & $195,3 \mathrm{AB}$ & $0,5 \mathrm{D}$ & $0,6 \mathrm{D}$ & $0,0 \mathrm{E}$ & $10,1 \mathrm{~B}$ & $42,2 \mathrm{E}$ & $86,9 \mathrm{BC}$ \\
\hline 4. Milho + Feijāo-guandu & $92,1 \mathrm{AB}$ & $185,4 \mathrm{ABC}$ & $1,4 \mathrm{D}$ & 2,6 DE & $3,9 \mathrm{DE}$ & $11,0 \mathrm{~B}$ & $61,3 \mathrm{DE}$ & $100,0 \mathrm{BC}$ \\
\hline 5. Milho + Mucuna-preta & $67,0 \mathrm{~B}$ & $145,6 \mathrm{ABCD}$ & D $5,3 \mathrm{AB}$ & $11,9 \mathrm{~B}$ & $24,8 \mathrm{AB}$ & $16,9 \mathrm{~A}$ & $110,9 \mathrm{CD}$ & $140,0 \mathrm{~B}$ \\
\hline 6. Milho + Labe-labe & $109,0 \mathrm{~A}$ & $184,0 \mathrm{ABC}$ & $3,5 \mathrm{C}$ & $5,8 \mathrm{BCD}$ & $6,9 \mathrm{CDE}$ & $11,5 \mathrm{~B}$ & $54,4 \mathrm{E}$ & $100,0 \mathrm{BC}$ \\
\hline \multicolumn{9}{|c|}{ Leguminosas Semeadas 21 d.a.s. ${ }^{1}$ Milho } \\
\hline 7. Milho + Crotalária & $26,1 \mathrm{C}$ & $57,4 \mathrm{BCD}$ & $0,7 \mathrm{C}$ & $1,5 \mathrm{D}$ & $4,3 \mathrm{CDE}$ & $10,9 \mathrm{~B}$ & $80,9 \mathrm{DE}$ & $233,3 \mathrm{~A}$ \\
\hline 8. Milho + Feijão-guandu & $31,0 \mathrm{C}$ & $44,0 \mathrm{CD}$ & $1,4 \mathrm{D}$ & 4,7 BCD & $18,6 \mathrm{BC}$ & $10,2 \mathrm{~B}$ & $174,9 \mathrm{AB}$ & $250,0 \mathrm{~A}$ \\
\hline 9. Milho + Mucuna-preta & $17,0 \mathrm{C}$ & $47,0 \mathrm{CD}$ & $6,3 \mathrm{~A}$ & $23,3 \mathrm{~A}$ & $34,9 \mathrm{~A}$ & $10,4 \mathrm{~B}$ & $156,8 \mathrm{ABC}$ & $280,0 \mathrm{~A}$ \\
\hline 10.Milho + Labe-labe & $15,0 \mathrm{C}$ & $30,5 \mathrm{CD}$ & $4,1 \mathrm{BC}$ & $12,9 \mathrm{~B}$ & $23,7 \mathrm{AB}$ & $10,4 \mathrm{~B}$ & $158,7 \mathrm{ABC}$ & $260,0 \mathrm{~A}$ \\
\hline F.Bloco & $2,71^{\mathrm{ns}}$ & $2,07^{\text {ns }}$ & $0,90^{\mathrm{ns}}$ & $2,05^{\mathrm{ns}}$ & $0,73^{\text {ns }}$ & $2,26^{\mathrm{ns}}$ & $2,36^{\mathrm{ns}}$ & $2,63^{\text {ns }}$ \\
\hline F.Tratamento & $31,61^{*}$ & $6,84^{* *}$ & $9,21 * *$ & $14,34^{* *}$ & $17,52^{* *}$ & $20,44^{* *}$ & $30,55^{* *}$ & $48,80^{* *}$ \\
\hline C.V. $(\%)$ & 19,7 & 40,7 & 8,9 & 44,5 & 36,5 & 7,3 & 16,9 & 12,4 \\
\hline d.m.s. & 34,0 & 143,2 & 1,6 & 10,1 & 14,4 & 2,5 & 52,5 & 67,3 \\
\hline
\end{tabular}

d.a.s. - dias após a semeadura

tas - plantas

Médias seguidas de mesma letra na coluna, nāo diferem estatisticamente entre si, pelo teste de Tukey $(\mathrm{P}>0,05)$.

is = năo significativo

* = significativo ao nível de $5 \%$ de probabilidade

espiga, resultado que difere do encontrado por Silva Neto (1981) em consórcio de milho + mucuna-preta em semeadura simultânea. Em valores absolutos o comprimento da espiga foi maior quando as leguminosas foram semeadas 21 dias após o milho. Neste caso o consórcio de milho com feijãoguandú destacou-se como o mais favorável. O efeito mais prejudicial ao comprimento de espiga foi observado para a semeadura simultânea de milho com labe-labe.

A altura das plantas de milho, a altura de inserção da primeira espiga e a produtividade do milho em monocultivo, mantido no mato, foram reduzidas em, respectivamente 13 , 24 e 36\%, em relação ao monocultivo no limpo (Tabela 2). As leguminosas, no sistema consorciado, em semeadura simultânea a do milho, também contribuíram para reduzir essas três variáveis. Comparando-se esse sistema ao monocultivo no limpo, houve redução de 13 a $18 \%$ na altura das plantas de milho, 22 a $28 \%$ na altura de inserção da primeira espiga e de 23 a $44 \%$ na produtividade, conforme a leguminosa utilizada. Nesse sistema, a mucuna-preta foi a que menos afetou o milho (Tabela 2).
Fleck et al. (1984) também verificaram redução na altura das plantas de milho devido à presença de plantas daninhas e do girassol semeado simultaneamente ao milho. Silva Neto (1981) observou redução na produção do milho quando em consórcio com mucuna-preta e labe-labe, mas não verificou efeitos negativos do feijão-guandu ou do mato, no milho em monocultivo. Entretanto, como já foi mencionado, a comunidade infestante deste trabalho era composta predominantemente por espécies de folha larga. Além disso, apresentava baixo número de indivíduos, o que causou pouca pressão competitiva. Ramalho et al. (1989) verificaram que no consórcio de milho com feijão, o mato que conviveu com a cultura do milho por todo ciclo determinou reduções de 23 a $73 \%$ no rendimento de grãos, dependendo do ano agrícola.

Conclui-se que a semeadura de leguminosas, 21 dias após a implantação da cultura do milho, reduziu a população de plantas daninhas e não interferiu na produtividade da cultura. Não houve efeito diferenciado entre crotalária, feijão-guandu, mucuna-preta e labe-labe sobre a comunidade infestante nem sobre o milho. Das leguminosas estudadas, as 
TABELA 2 - Interferência das plantas daninhas e leguminosas sobre algumas variáveis de produção do milho. Cascavel/PR, 1989/90.

\begin{tabular}{|c|c|c|c|c|c|}
\hline Tratamento & $\begin{array}{l}\text { Comprimento } \\
\text { de espiga (cm) }\end{array}$ & $\begin{array}{l}\text { Peso de } 100 \\
\text { grãos }\end{array}$ & $\begin{array}{c}\text { Altura de } \\
\text { plantas (M) }\end{array}$ & $\begin{array}{c}\text { Altura de } \\
\text { inserçāo da 1: } \\
\text { espiga (m) }\end{array}$ & $\begin{array}{l}\text { Produtividade } \\
\text { (kg/ha) }\end{array}$ \\
\hline 1. Milho no limpo & $14,8 \mathrm{AB}$ & 33,8 & $2,39 \mathrm{~A}$ & $1,47 \mathrm{~A}$ & $7.133 \mathrm{~A}$ \\
\hline 2. Milho no mato & $12,8 \mathrm{BC}$ & 33,1 & $2,08 \mathrm{BCD}$ & $1,11 \mathrm{~B}$ & $4.581 \mathrm{CD}$ \\
\hline \multicolumn{6}{|l|}{ Semeadura Simultânea } \\
\hline 3. Milho + Crotalária & $12,5 \mathrm{BC}$ & 30,0 & $2,07 \mathrm{CD}$ & $1,12 \mathrm{~B}$ & $4.139 \mathrm{CD}$ \\
\hline 4. Milho + Feijẫo-guandu & $12,3 \mathrm{BC}$ & 30,9 & $1,96 \mathrm{D}$ & $1,09 \mathrm{~B}$ & $4.358 \mathrm{CD}$ \\
\hline 5. Milho + Mucuna-preta & $12,4 \mathrm{BC}$ & 30,6 & $2,07 \mathrm{CD}$ & $1,14 \mathrm{~B}$ & $5.505 \mathrm{BC}$ \\
\hline 6. Milho + Labe-labe & $11,0 \mathrm{C}$ & 30,6 & $1,99 \mathrm{D}$ & $1,06 \mathrm{~B}$ & $4.006 \mathrm{D}$ \\
\hline \multicolumn{6}{|c|}{ Leguminosas Semeadas 21 d.a.s. ${ }^{1}$ Milho } \\
\hline 7. Milho + Crotalária & $13,7 \mathrm{ABC}$ & 31,0 & $2,31 \mathrm{~A}$ & $1,41 \mathrm{~A}$ & $6.445 \mathrm{AB}$ \\
\hline 8. Milho + Feijão-guandu & $15,6 \mathrm{~A}$ & 31,0 & $2,27 \mathrm{ABC}$ & $1,36 \mathrm{~A}$ & $7.249 \mathrm{~A}$ \\
\hline 9. Milho + Mucuna-preta & $14,6 \mathrm{ABC}$ & 31,7 & $2,28 \mathrm{ABC}$ & $1,37 \mathrm{~A}$ & $6.492 \mathrm{AB}$ \\
\hline 10.Milho + Labe-labe & $14,6 \mathrm{ABC}$ & 31,2 & $2,30 \mathrm{AB}$ & $1,40 \mathrm{~A}$ & $7.094 \mathrm{~A}$ \\
\hline F.Bloco & $0,51^{\mathrm{ns}}$ & $0,93^{\mathrm{ns}}$ & $0,92^{\mathrm{ns}}$ & $0,27^{\mathrm{ns}}$ & $0,23^{\mathrm{ns}}$ \\
\hline F.Tratamento & $5,90^{* *}$ & $0,99^{\mathrm{ns}}$ & $12,88^{* *}$ & $20,77^{* *}$ & $22,94^{* *}$ \\
\hline C.V. $(\%)$ & 6,8 & 5,4 & 3,4 & 5,0 & 8,5 \\
\hline d.m.s. & 2,7 & 4,9 & 0,21 & 0,18 & 1,410 \\
\hline
\end{tabular}

1 d.a.s. - dias após a semeadura

Médias seguidas de mesma letra na coluna, náo diferem estatisticamente entre si, pelo teste de Tukey $(\mathrm{P}>0,05)$.

$\mathrm{ns}=$ nảo significativo

$\bullet$ = significativo ao nível de $5 \%$ de probabilidade

três últimas são as mais promissoras. Exclui-se a crotalária por apresentar dificuldade em se estabelecer no sistema consorciado. A critério do agricultor, dependendo da finalidade e do custo de produção, a mucuna-preta é opção pare o consórcio em semeadura simultânea do milho.

\section{LITERATURA CITADA}

BHARDWAJ, S.P., PRASAD, S.N., SINGH, G. Economizing $\mathrm{N}$ by greem manure in rice wheat rotation. Indian Journal of Agriculture Science, v.52, p.86-90, 1981.

CARVALHO, H.W.L. Cultivares de miho e de feijão em monocultivo consorciado. I. Ensaios de rendimentos. Pesquisa Agropecuária Brasileira, v.25, n.7, p.10031010, 1990.

CHOWDHURY, M. K., ROSARIO, E. L. Phosphorus utilization efficency as affected by component population, rizobial inoculation and applied nitrogen in maize, mungbean intercropping. Experimental Agriculture, v.28, p.255-263, 1992.
DALAL, R.C. Effects of intercropping maize with pigeon-pea on grain yield and nutrient uptake. Experimental Agriculture, v.10, n.3, p.219-224, 1974.

FLECK, N.G., MACHADO, C.M.N., SOUZA, R.S. Eficiência da consorciação de culturas no controle de plantas daninhas. Pesquisa Agropecuária Brasileira, v.19, n.5, p.591-598, 1984.

FLESCH, R.D., ESPÍNDULA, E.A. Cultivares de feijão para consórcio com milho. Pesquisa Agropecuária Brasileira, v.22, n.7, p.699-704, 1987.

KUMAR RAO, J.V.D.K., DART, P.J., SASTRY, P.V.S.S. Residual effect of pigeonpea (Cajanus cajam) on yield and nitrogen response of maize. Experimental Agriculture, v.19, p.131-141, 1983.

LIMA, J.M.P., LIMA, L.A.P., ANDRADE, L.A.B., REZENDE, P.M. Influência de diferentes populações de plantas e da adubação no sistema consorciado milho e feijão. Pesquisa Agropecuária Brasileira, v.22, n.8, p.817-823, 1987. 
MACHADO, C.M.N., FLECK, N.G., SOUZA, R.S. Comportamento dos componentes do rendimento de cultures consorciadas. Pesquisa Agropecuária Brasileira, v.19, n.8, p.985-997, 1984.

MARTINS, D., PITELLI, R.A. Effects of liming and phosphorus fertilization on interspecific interference between soybean and alexandergrass [Brachiaria plantaginea (Link) A.S. Witchc.] under greenhouse conditions. In: MEETING OF WEED SCIENCE SOCIETY OF AMERICA, 33, Denver/Colorado, 1993. Abstracts. Champaign: WSSA, 1993, p.48.

NAIR, K.P.P., PATEL, U.K., SINGH, R.P., KAUSHIK, M.K. Evaluation of legume intercropping in conservation of fertilizer in maize culture.Jounal of Agriculture Science, v.93, p.189-194, 1979.

PANDEY, R.K., PENDLETON, J.W. Soybeans as green manure in a maize intercropping system. Experimental Agriculture, v.22, p.179-185, 1986.

RAMAKRISHNA, A., ONG, C.K., REDDY, L.N. Canopy duration and structure of pigeonpea intercropped with upland rice. Experimental Agriculture, v.17, p.311$315,1992$.
RAMALHO, M.A.P., CRUZ, J.C., PASSINI, T. Competição de plantas daninhas nas culturas do milho e feijão consorciados. Pesquisa Agropecuária Brasileira, v.24, n.5, p.543-552, 1989.

RAMALHO, M.A.P., SILVA, A.F., AIDAR, H. Cultivares de milho e feijão em monocultivo e em dois sistemas de consorciação. Pesquisa Agropecuária Brasileira, v.19, n.7, p.829-833, 1984.

REIS, W.P., RAMALHO, M.A.P., CRUZ, J.C. Arranjos e populações do feijoeiro na consorciação com milho. Pesqui sa Agropecuária Brasileira, v. 20, n. 5, p.575-584, 1985.

SILVA NETO, B. Avaliação da possibilidade do cultivo múltiplo de milho e leguminosas e os efeitos sobre o desenvolvimento da comunidade infestante de plantas daninhas. Jaboticabal: FCAV/UNESP, 1981. 34 p. Trabalho de Graduação em Agronomia.

YADAV, R.L. Intercropping pigeonpea to conserve fertilizer nitrogen in maize and produce residual effects on sugarcane. Experimental Agriculture, v.17, p.311315,1981 